\title{
Discusiones de metodología La observación en la investigación social: la observación participante como construcción analítica
}

\author{
Francisca Fernández Droguett
}

\begin{abstract}
Resumen
Este trabajo tiene como propósito reflexionar sobre la importancia de la observación en la investigación social, cuestionando la idea de que la observación constituye una práctica objetiva neutral. La objetividad responde a una construcción analítica, situada en los marcos de referencia del observador, siendo siempre la observación una interpretación determinada por el contexto de producción de conocimiento, que parte de la realidad pero cuestionándola, convirtiendo el hecho en dato.
\end{abstract}

Palabras clave: Observación, Observación participante, Observación exógena, Observación endógena, Auto-observación.

\begin{abstract}
This essay has the goal of thinking about the importance of the observation in the social research through the critics of the idea that observation constitute a neutral objective practice. The objectivity answers to an analytic construction placed within the reference background of the observer. In this sense, the observation is always an interpretation determined by the context of knowledge production which comes from the reality but questionning it and changing the fact in a piece of information.
\end{abstract}

Key words: Observation, Participant observation, Exogenous observation, Endogenous observation, Self observation.

* Chilena. Antropóloga y Magíster en Psicología Social. Doctorante en Estudios Americanos, mención Pensamiento y Cultura, Instituto de Estudios Avanzados de la Universidad de Santiago de Chile. Docente de la Universidad ARCIS y la Universidad Internacional SEK. Correo electrónico: franciscafernandez@esfera.cl. Fono: 4745564. 
La observación en la investigación social: la observación participante como construcción analítica - Francisca Fernández Droguett

\section{Introducción}

La observación es una de las actividades comunes de la vida diaria, siendo parte de la experiencia sensorial de los sujetos; a través del sentido de la vista conocemos el mundo. La información que se presenta a través de la observación nos resulta evidente pero también es incompleta, por lo que puede transformarse en una poderosa herramienta de investigación social sólo si se trasciende el orden de lo evidente, enfocándola a un objeto concreto de investigación, planificándola en fases, aspectos, lugares y personas.

Observar no es lo mismo que mirar, ya que la observación supone un posicionamiento y una construcción de la mirada, siendo una mirada que construye y produce la realidad observada. Cuando observamos interrogamos, indagamos, cuestionamos lo observado. Observar es "examinar atentamente... advertir, reparar ... mirar con atención." (Diccionario de la Real Academia Española).

La ciencia parte de la observación, pero ¿qué hace que una observación pueda llegar a ser científica? Cuando la observación supone una metodología, una construcción de la mirada, cuando constituye una forma de conocimiento sistemático de la realidad, estamos ante una observación "científica". Observar es elegir, es clasificar, es aislar en función de las teorías.

\section{La observación en la actividad científica}

Desde una concepción científica ingenua se ha pensado que las teorías científicas derivan de los hechos de la experiencia adquiridos mediante la observación. La ciencia es objetiva y fiable, es un conocimiento objetivamente probado, cumpliendo el observador científico la función de registrar de un modo fidedigno lo observado. Sin embargo para Chalmers (2001) la observación no es suficiente para comprobar los resultados de ésta, siendo necesario un conocimiento enraizado en un conjunto de relaciones 
entre teorías. Cada observación implicaría una teoría. Existe la realidad en cuanto tal, pero posee diversos niveles, y por ende, múltiples interpretaciones.

De igual modo, para Durkheim (2000) el conocimiento inmediato, directo, las prenociones no constituyen la base para un conocimiento científico, sino más bien en su negación sistemática se consolida el quehacer científico. La observación de la realidad responde a una construcción analítica, donde el objeto de estudio no estaría dado, se construye analíticamente como objetividad en tanto entidad coherente a las premisas estipuladas por el investigador.

Para otro autor clásico, Weber (1997), lo que se conoce de la realidad son parcialidades, renunciando a la idea de realidad absoluta. La observación en Weber es una observación comprensiva-explicativa, que da forma a las relaciones sociales como acciones de sentido. Desde esta perspectiva la construcción del objeto de estudio deviene en la construcción de tipos ideales, en tanto relaciones posibles, a través de una explicación causal de las acciones humanas. El sujeto cognoscente observa la realidad de modo parcial, a través de la aplicación de tipos ideales, por lo que la objetividad proviene de la interpretación y aplicación de estos tipos. Se comprende la realidad a través de una abstracción desde los rasgos típicos concebidos como modelos de comportamiento racionales.

"Se pueden observar en la acción social regularidades de hecho; es decir, el desarrollo de una acción repetida por los mismos agentes o extendida a muchos (...), cuyo sentido mentado es típicamente homogéneo. La sociología se ocupa de estos tipos de desarrollo de la acción" (Weber 1997: 23).

La realidad es un campo de posibilidades, donde toda observación se encuentra inmersa en diversos planos de realidad. La observación no da cuenta de la totalidad de la realidad, sino más bien de una parcialidad del mundo de lo social. El objeto no está 
La observación en la investigación social: la observación participante como construcción analítica - Francisca Fernández Droguett

dado, por lo que la única forma de conocer la realidad es transgrediendo los límites propios del sujeto cognoscente, mediante la construcción de categorías analíticas que permitan al investigador abrirse a nuevas experiencias.

Siguiendo este planteamiento, Maffesoli (1993) piensa la realidad como polisémica, concibiendo al sujeto como una perspectiva, una mirada. Para observar la realidad se requiere de teorías que se cuestionen constantemente, de un diseño de investigación flexible, que se adecue a las dinámicas propias del mundo social, incorporando una multiplicidad de puntos de vista en torno a un mismo hecho, pretendiendo únicamente llegar a verdades aproximativas.

“En efecto, la misma situación acepta diferentes puntos de vista y, se quiera o no, esta multiplicidad es la negación de facto del concepto eterno y de las diversas explicaciones en última instancia... nuestra definición de los conceptos depende de nuestra posición y de nuestro punto de vista" (Maffesoli 1993: 159).

Por ende, la observación como método o técnica de investigación científica supone una función indagadora por la cual se interroga la realidad; constituye una práctica permanente de búsqueda del sentido de las cosas observadas, organizando e introduciendo una serie de recortes para analizar un campo determinado. Debemos ir más allá de la realidad de lo observado, de lo evidente, entendiendo que toda observación es una construcción analítica a través de la cual explicamos e interpretamos la realidad, seleccionando un ámbito específico del campo social. La observación es una perspectiva, constituye un punto de vista. No obstante, cada hecho observado supone un conjunto de significados, una normatividad social, que el observador deberá interpretar.

Toda observación representa una interpretación, es parte de un esquema o marco de referencia social que le da sentido. Los sujetos interiorizan individualmente un conjunto de relaciones 
sociales, esquemas de distinción, como objetividad colectiva, lo que produce un determinado tipo de lectura de lo social.

“El proceso de la observación no se reduce sólo a sensaciones y percepciones sino también implica la presencia activa de esquemas teóricos que permiten seleccionar el dato que viene de la realidad. Segundo, la dinámica de la observación no es una simple lectura del dato mismo sino que, principalmente, tiene significación en las estructuras teóricas previas en las cuales se inserta y cobra sentido. Se observa la realidad externa como una interpretación estructurada que posee significación" (Mejía 2002: $\mathrm{s} / \mathrm{p})$.

\section{La observación participante como observación exógena}

Desde las Ciencias Sociales, la modalidad más conocida de observación sistemática es la observación participante. Este término se ha usado en la investigación sociológica y antropológica para designar una estrategia metodológica compuesta por una serie de técnicas de obtención y análisis de datos, entre las que se incluye la observación y participación en el lugar de los hechos. Para Denzin:

"Hay una curiosa mezcla de técnicas metodológicas en la observación participante: se entrevistará a gente, se analizarán documentos del pasado, se recopilarán datos censales, se emplearán informantes y se realizará observación directa. Para los propósitos presentes la observación participante será definida como una estrategia de campo que combina simultáneamente el análisis de documentos, la entrevista a sujetos e informantes, la participación y observación directa, y la introspección" (Denzin 1970: 185-186).

Para diversos autores (Sánchez, 2004; Taylor y Bodgan, 1992) la observación participante se define como un método de investigación que implica la inserción del investigador en el lugar de la 
La observación en la investigación social: la observación participante como construcción analítica - Francisca Fernández Droguett

observación, suponiendo una mirada distante, lejana, extraña del observador hacia lo observado. El sujeto se inserta en la realidad observada, pero siempre como un intruso. En este sentido, toda observación participante constituye una observación externa, ya que el distanciamiento del investigador frente a lo observado garantizaría la confiabilidad y validez de su registro.

"Recomendamos que los investigadores se abstengan de estudiar escenarios en los cuales tengan una directa participación personal o profesional... Cuando uno está directamente involucrado en un escenario, es probable que vea las cosas desde un solo punto de vista. En la vida cotidiana, las personas asumen modos sobrentendidos de ver las cosas, y equiparan lo que ven con la realidad objetiva" (Taylor y Bodgan 1992: 36).

Desde esta visión, se aconseja abstenerse de observar escenarios o a sujetos con quienes el investigador posee algún tipo de cercanía personal o profesional. Nunca se debería seleccionar un escenario demasiado conocido, esto impediría dilucidar lo latente, lo oculto a simple vista. Bajo este supuesto la observación participante es una observación exógena, pero al mismo tiempo cercana, que implica necesariamente el involucramiento del investigador con lo observado, para comprender el conjunto de símbolos y significados compartidos por los sujetos.

Kenneth L. Pike (1971) y Marvin Harris (1991) establecen dos términos diferenciatorios, lo etic y lo emic, como dos momentos constitutivos (e interdependientes) del quehacer investigado para la comprensión de la realidad observada. El término etic ("fonetic") se refiere a la percepción que tiene un observador experimentado de los datos que recoge situándose desde fuera de la cultura que observa, fijando contenidos culturales a partir de factores que no son percibidos por los miembros del grupo en estudio. En cambio, desde un punto de vista emic ("fonemic"), el observador trabaja con las coordenadas socio-culturales del grupo observado, reproduciendo los contenidos culturales de los 
sujetos investigados según los propios marcos de referencia de éstos.

Una de las críticas que podemos plantear a este marco diferenciatorio es que todavía opera la idea de un investigador como un sujeto externo de la realidad observada, integrándose su punto de vista exclusivamente como un experto de la observación, un científico de la mirada, y no como un sujeto que forma parte, a su vez, de la realidad observada. Del mismo modo, se niega la posibilidad que los propios informantes tengan conocimiento de un conjunto de categorías provenientes del ámbito científico.

Para comprender y explicar los aspectos más significativos de lo social, se requiere del rapport del investigador con sus informantes. El concepto posee una multiplicidad de definiciones, se le puede entender como una forma de compartir con el mundo simbólico de los informantes, como empatía, como apertura de la gente hacia el investigador, como una estrategia para que la gente vea al investigador como intachable, como una manera de penetrar, irrumpir en la gente, siendo unos de sus requisitos que el observador se acomode a las prácticas rutinarias de los sujetos (Taylor y Bodgan 1992).

En el establecimiento del rapport, el investigador se relaciona con sujetos determinados, quienes cumplirán la labor de facilitar la llegada, el acceso y la comprensión del campo. Esos sujetos serán los informantes claves de la investigación. Ellos proporcionarán una comprensión profunda del escenario en cuestión, a partir de su comprensión interna, en tanto parte de la comunidad observada. Pero no debemos olvidar que todo sujeto interpreta la realidad de un determinado modo, por lo que la selección de ciertos informantes implica ya una mirada particular, subjetiva, a partir de la posición que ocupa el informante en el entramado social. Del mismo modo toda interpretación es intersubjetiva, se construye a partir de una serie de referencias sociales en relación a otros sujetos. 
La observación en la investigación social: la observación participante como construcción analítica - Francisca Fernández Droguett

El informante clave se constituye como observador del observador, observa su entorno a la luz de sus propias categorías como también a través de las categorías científicas del investigador, a través de la articulación de una doble hermenéutica. El investigador interpreta la acción de los otros, y a su vez los investigados interpretan su propia acción a partir de la interpretación del investigador, con lo que toda actividad científica está inserta en una doble hermenéutica, y toda metodología debe adecuarse en términos de una realidad compleja. Para Giddens (1987) se deben considerar tanto los componentes interpretativos de la acción como los funcionales, entendiendo lo interpretativo en términos empáticos, recogiendo los significados de los propios actores en tanto reflexividad sobre su propia experiencia.

\section{Observación de primer y segundo orden en la observación participante}

En el proceso de recolección-construcción de datos significativos, para Rolando Sánchez (2004), el observador debe cumplir algunas condiciones metodológicas para la observación. Debe ser extranjero, o en términos más específicos, debe ser un extraño para la comunidad; debe convivir, participar del diario vivir de los informantes; debe mantener siempre la distancia con el objeto, involucrarse pero hasta cierto punto; la observación debe ser reflexiva y crítica de los procesos sociales. Por lo tanto, se puede deducir que la observación participante correspondería a una observación de segundo orden, donde el investigador es un observador especializado que observa a otros observadores cotidianos y las significaciones de las observaciones de primer nivel, mediante un conjunto de herramientas teórico-metodológicas especializadas.

Para entender este punto se debe señalar qué se entiende por observación de primer y segundo orden. En una observación de primer orden los actores se desenvuelven en un mundo cotidiano, inmediato, observándose mutuamente como objetos, donde 
no pueden observar el marco de distinción que hace posible la observación. Desde esta visión la realidad permanece latente para quienes participan de ésta, siendo la única manera de trascender lo obvio, rescatando lo oculto ante la mirada del "nativo", la presencia de un observador externo. Por lo que la observación participante es necesariamente una observación exógena, de segundo orden. El investigador tiene la posibilidad de ver lo que los otros, sujetos cotidianos, no pueden ver.

“La observación de primer orden implica sujetos cotidianos que reflexionan sobre la realidad, tienen un conocimiento de sentido común de la vida social y de acuerdo a ello actúan sobre la propia sociedad. Los sujetos de primer orden son observadores que procesan información y generan conocimiento que les permite relacionarse en la sociedad" (Mejía 2002: s/p).

En la observación de segundo orden el investigador busca comprender por qué el investigado observa como observa, siendo una forma especializada que no sólo observa a un objeto distinguible sino que también busca captar los esquemas de distinción que realizan los observadores de primer orden en su mundo cotidiano, a través de su propio esquema de conocimiento de la realidad social. En este nivel de observación, el investigador tiene la capacidad de comparar y articular las diversas observaciones de los sujetos combinando un conjunto de distinciones provenientes de sus informantes. El investigador es capaz de reconstruir teóricamente la realidad social, buscando con este procedimiento los significados latentes y sus estructuras.

"Tal clase de observación (la de segundo orden) directa lleva a explicarse por qué el otro observador observa como observa. Así, los afectados esbozan teorías propias acerca de la conducta de riesgo de quienes deciden, y quienes deciden esbozan teorías propias acerca de la conducta de protesta de los afectados. Así se obtienen experiencias, y ciertamente existen posibilidades de refinar y mejorar tales explicaciones, de equipararlas con una 
La observación en la investigación social: la observación participante como construcción analítica - Francisca Fernández Droguett

mayor complejidad y con posibilidades de comunicación más exactas" (Luhman 1998: 285).

\section{La observación participante como mirada endógena}

Para Guasch (1997), a diferencia de otros autores, la observación participante corresponde a una técnica de investigación social para estudiar y analizar la vida social desde dentro, siendo necesario que el investigador se convierta en el nativo. Desde su punto de vista, las personas ajenas al mundo social investigado tienden a definir la realidad de manera simple y cartesiana. En cambio, los miembros de un colectivo observado elaboran conceptos más complejos que el investigador, por ello la importancia de incorporar sus puntos de vista a través de una mirada cercana, próxima del investigador hacia lo observado. La observación participante como observación exógena, distante, es un intento de neutralidad política, de no involucramiento. La proximidad al fenómeno es lo que garantizaría el acceso al campo de investigación, mediante el establecimiento de una relación sujeto-sujeto entre investigador e investigado, desde una pretensión dialógica.

Michail Bajtin (1995), define como dialogicidad la posibilidad de comprender el enunciado de otra persona, orientarse en relación a él, estableciendo su lugar dentro del contexto correspondiente. En este sentido, todo discurso, todo enunciado se orienta no sólo hacia otras personas sino también se relaciona con otros discursos, otros enunciados. Por ello toda relación dialógica implica intertextualidad, donde cada texto, discurso, palabra, es un lugar de cruce de múltiples textos, discursos, palabras desde donde se produce sentido, siendo un proceso comunicativo socialmente situado.

A partir de esta definición Paulo Freire (1968) define dialogicidad como una relación por construir de diálogo entre los sujetos partícipes de una dinámica social, siendo un concepto que va 
más allá del diálogo, implica acción, reflexión y un proceso constante de transformación social para la construcción de relaciones sociales equitativas. En la relación dialógica entre el investigador y el sujeto investigado, tanto el lugar del investigador como de los sujetos en estudio, son espacios donde se problematizan las relaciones de poder. No se trata de una relación ingenua, sino más bien de una práctica social problematizadora, donde los sujetos ocupan posiciones desde el cruce de relaciones múltiples.

\section{La autoobservación}

En contraste, como procedimiento inverso a la observación participante, se encuentra la autoobservación, considerada una de las modalidades posibles de observación endógena, propia de sistemas observadores. La distinción aludida gira en torno a las nociones de observación exógena - observación endógena, y a los conceptos de sistema observado - sistema observador procedentes de la cibernética.

“... los conceptos de sistema observado y sistema observador... representan [en el campo de la cibernética] el paso de una teoría del control del comportamiento y la comunicación en animales y máquinas a una autoorganización y complejización creciente de los sistemas. (...) La autoobservación constituye un procedimiento de aprendizaje/conocimiento inverso del realizado en la observación participante: en lugar de aprender a ser un nativo de una cultura extraña (en lugar de ser un observador externo que pretende un estado de observador participante), el nativo aprende a ser un observador de su propia cultura..." (Delgado y Gutiérrez 1994:162-163).

Para autores como Miguel Martínez (2004) y Oscar Guasch (1997), los miembros de una cultura o un grupo especial comparten una estructura propia subyacente de razonamiento, lo que facilitaría la auto-interpretación, ya que manejarían la estructura básica de su propio pensamiento. 
La observación en la investigación social: la observación participante como construcción analítica - Francisca Fernández Droguett

"Se parte de la idea de que los miembros de esos grupos están en posición privilegiada para estudiar sus problemas... Si un grupo étnico, cultural o situacional tiene una estructura lógica y de pensamiento propia, tiene, por eso mismo, una teoría del conocimiento, una epistemología especial" (Martínez 2004: 229).

Para Mari Luz Esteban (2004) la autoobservación constituye un procedimiento de aprendizaje/conocimiento donde el observador se observa a sí mismo, produciéndose un proceso reflexivo en torno a sus visiones, sus formas de vida, sus comportamientos y acciones, frente a lo observado. Se parte de la propia experiencia, del lugar del sujeto observador como testigo y narrador, reconociéndose como actor social. El lugar del investigador se cruza con el lugar del investigado. En la autoobservación, la subjetividad se convierte en una forma de conocimiento para la investigación social, partiendo de la vivencia más cercana, la del propio investigador.

Una de las principales críticas que se realiza a esta modalidad de autoobservación es el supuesto exceso de individualización y personalización. Pero justamente, a partir de esta situación, a través de la autoobservación el investigador actúa sobre sí mismo, se autoexamina, reflexiona sobre el modelo interpretativo que posee y autorreflexiona sobre los propios conocimientos que va construyendo, como una forma de garantizar una mayor objetividad a partir de constatar desde qué lugar se está hablando.

"El investigador no es sujeto pasivo y externo al proceso, que sólo se limita a registrar la información, es un agente activo, una forma de acción, tiene la capacidad de controlar y dar sentido a sus observaciones según criterios de distinción y, además, posee la facultad de autoobservación sobre sus propias observaciones, de reflexionar sobre sus propios pensamientos. En ese sentido, el investigador social es un sujeto en proceso que se construye en la misma actividad cognoscitiva, en interacción estrecha con su objeto, los sujetos de primer orden" (Mejía 2002. s/p). 


\section{Objetividad y subjetividad en la observación participante: hacia la construcción de un enfoque pluralista}

A mi modo de ver, tanto en la observación participante como en la auto-observación el investigador, en tanto inmerso en las tramas sociales que estudia, no es ajeno a lo que investiga y por lo tanto tampoco puede ser neutro. El investigador está implicado en la vida social y por eso debe asumir su rol como crítico social. El observador, con su sola presencia, modifica la realidad, pero además jamás puede trascender su propio mundo, por lo que siempre observa desde un determinado lugar, por ello ningún investigador social puede renunciar totalmente al cartesianismo, ya que proviene de un mundo, el académico, donde la práctica investigativa se ha basado históricamente en este tipo de racionalidad científica.

Por ende toda observación es subjetiva (Guasch 1997), la situación social del observador condiciona aquello que ve y mira. Pero a su vez, la situación de quien observa mediatiza tanto la observación como la interpretación y las relaciones de campo.

No podemos negar que la observación es subjetiva en tanto mirada situada, pero la observación en la investigación social es del mismo modo objetiva. En la medida que reconoce y explicita el observador su posición social la subjetividad queda matizada. Del mismo modo la articulación e incorporación de diversos puntos de vista, a través de una metodología pluralista, puede constituir un criterio de objetividad.

Para Donna Haraway (1995) en las diversas Ciencias se ha asumido únicamente dos formas de conocer la realidad: la objetividad y el relativismo. El problema es que la objetividad se ha erigido como el único conocimiento válido, siendo una mirada desde todos los lugares a la vez, y el relativismo asume que toda posición y construcción es potencialmente válida, produciendo conocimiento desde ningún lugar en particular. 
La observación en la investigación social: la observación participante como construcción analítica - Francisca Fernández Droguett

A diferencia de ambos planteamientos, Haraway propone un conocimiento generado desde posiciones determinadas, sitios localizables, por "lugares" que ocupamos en diferentes espacios sociales, posiciones entendidas como productos, en las cuales habitamos y desde las cuales se ven distintas realidades. Somos responsables de lo que pensamos y decimos; asumimos nuestro rol activo en la investigación, asumimos que no podemos mirar desde afuera, siempre nos involucramos con lo observado.

"La alternativa al relativismo son los conocimientos parciales, localizables y críticos, que admiten la posibilidad de conexiones llamadas solidaridad en la política y conversaciones compartidas en la epistemología. El relativismo es una manera de no estar en ningún sitio mientras se pretende igualmente estar en todas partes. La "igualdad" del posicionamiento es una negación de responsabilidad y de búsqueda crítica. El relativismo es el perfecto espejo gemelo de la totalización en las ideologías de la objetividad. Ambos niegan las apuestas en la localización, en el encarnamiento y en la perspectiva parcial, ambos impiden ver bien... La moraleja es sencilla: solamente la perspectiva parcial promete una visión objetiva" (Haraway 1995:329).

A esta forma de conocer Haraway la llama "objetividad encarnada", siendo una objetividad que se basa en una localización limitada y en cocimientos situados, parciales. Cuando habla de parcialidad se está refiriendo a la parcialidad de la mirada, a un tipo de conocimiento encarnado donde la posición define las posibilidades de lectura y acción, nuestro posicionamiento para el diálogo con lo otro.

Una metodología situada de investigación implica la construcción del "objeto" asumiendo un punto de vista desde el cual se interpretan y viven las cosas. Por lo tanto, jamás conocemos la realidad en su totalidad, ya que no se puede abarcar todo, sólo conocemos una parcialidad del fenómeno investigado. 
Wallerstein (1997) plantea que otra forma de pensar la objetividad es concibiéndola como una posibilidad de abrir e incorporar diversos horizontes interpretativos, por lo tanto la objetividad se enraíza en universales parciales y no en un solo universal. La inclusión de diversos mundos interpretativos recupera e integra la reflexividad como actividad sistemática en la construcción del fenómeno. La inclusión de las formas de conocimiento de los actores sociales se torna en un elemento central en la construcción de un conocimiento más objetivo, como apertura a otros interlocutores en tanto otras experiencias culturales. Pero no olvidemos que el autor propone un universalismo pluralista, que incorpore múltiples visiones de mundo pero siempre teniendo en consideración escalas de valores comunes y universales. La objetividad no es un conocimiento a priori, es el resultado de un aprendizaje humano, y su meta es constituirse en una objetividad intercultural, de diálogo entre diversas culturas.

“Es preciso responder simultáneamente a las demandas de relevancia (aplicabilidad, validez) universal y reconocer a la vez la continuada calidad de una multiplicidad de culturas;... Las ciencias sociales deberían emprender un proceso de apertura muy amplio hacia la investigación y la enseñanza de todas las culturas en la búsqueda de un universalismo pluralista renovado, ampliado y significativo" (Wallerstein 2001: 97).

\section{A modo de cierre}

Considerando lo anteriormente expuesto, podemos decir que la observación participante es una situación en la cual el observador queda comprometido en el juego de todas las miradas. En la participación el observador se despoja no de la objetividad de la observación sino de la neutralidad distante del espectador. Pero la participación no debe ser entendida únicamente como una intervención en un determinado lugar, sino como una forma de apropiación e identificación con los sentidos, los significados de los sujetos observados. 
La observación en la investigación social: la observación participante como construcción analítica - Francisca Fernández Droguett

Más allá de recopilar datos, a través de la observación producimos la información pertinente para la problemática a analizar. Lo que construimos son las interpretaciones de los hechos y nos los hechos en sí mismos, construimos los datos para interrogar la realidad, mediante su complejización. En palabras de Marx (1997) "pasamos desde lo concreto-concreto a lo concretopensado". Lo concreto es una síntesis de una serie de múltiples relaciones. En un primer nivel, esta multiplicidad de relaciones se encuentran en una situación de desorden (se expresa como concreto-concreto), por ello se hace necesario un proceso de abstracción para poder ordenar. El proceso de pasar al concreto pensado desde lo concreto da orden y categoriza, constituye un proceso encaminado a complejizar las relaciones que a primera vista aparecen como caóticas, mediante la elaboración de categorías que permitan captar lo concreto a partir de relaciones constituyentes como totalidad concreta.

La observación participante posee la pretensión de dar cuenta de cómo la realidad observada es vivida por los sujetos, quienes le confieren un sentido propio y particular. Ningún fenómeno tiene un significado inherente, por lo que en sí no constituye un dato sino simplemente una fuente potencial para la construcción del dato. Se convierte en un dato al relacionarse con una práctica científica, que selecciona entre diversos aspectos los que son significados y pertinentes a la problematización de la investigación. Lo observado debe ser siempre pensado, y por ello es construido.

La construcción del hecho en dato nos da cuenta de que la observación participante está desprovista de toda espontaneidad, la problematización fija qué se va a observar y de qué modo.

\section{Bibliografía}

Bajtin, Mikhail (1995). La estética de la creación verbal, México, Siglo XXI.

Chalmers, Alan (2001). ¿Qué es esa cosa llamada ciencia?, México, Siglo XXI. 
Delgado y Gutiérrez (1994). Métodos y técnicas cualitativas de investigación en Ciencias Sociales, Madrid, Síntesis Psicológica.

Denzin, Norman K. (1970). The research act: a theoretical introduction to sociológical Methods, Chicago, Aldine Publishing Company.

Durkheim, Emile (2000). Las reglas del método sociológico, México, Quinto Sol.

Esteban, Mari Luz (2004). Antropología encarnada. Antropología desde una misma. Consultado el 24, 05, 2006 en http:/ / www.ehu.es/CEIC/ pdf/12.pdf

Freire, Paulo (1968). La pedagogía del oprimido, México, Siglo XXI.

Giddens, Anthony (1987). Las nuevas reglas del método sociológico, Buenos Aires, Amorrortu.

Guasch, Oscar (1997). Observación participante, Madrid, Centro de Investigaciones sociológicas.

Haraway, Donna (1995). Ciencia, cyborgs y mujeres. La reinvención de la naturaleza, Madrid, Ediciones Cátedra.

Harris, Marvin (1991). Emic, etic y la nueva etnografía. En: El desarrollo de la teoría antropológica, Madrid, Taurus, pp. 419-495.

Luhmann, Niklas (1998). La observación de segundo orden. En: Sociología del riesgo, México, Triana, pp. 273-285.

Maffesoli, Michel (1993). El conocimiento ordinario. Compendio de sociología, México, FCE.

Martínez, Miguel (2004). Arte y Ciencia en la Metodología Cualitativa, México, Trillas.

Marx, Karl (1997). Introducción general a la crítica de la economía política/ 1857, México, Siglo XXI.

Mejía, Julio (2002). Perspectiva de la Investigación Social de Segundo Orden. Consultado el 3, 06, 2006, en http://www.moebio.uchile. $\mathrm{cl} / 14 /$ mejia.htm

Pike, Kenneth L. (1971). Etic and Emic Standpoints for the Description of Behavior. En: Language in Relation to an Unified Theory of the Structure of Human Behavior, Paris, The Hague, pp. 8-12.

Sánchez, Rolando (2004). La observación participante como escenario y configuración de la diversidad de significados. En: Observar, escuchar y comprender. Sobre la tradición cualitativa en la investigación social, editado por M. Tarrés. México, El Colegio de México/ Porrúa, pp. 97-131. 
La observación en la investigación social: la observación participante como construcción analítica - Francisca Fernández Droguett

Taylor, S. J., y Bodgan, R. (1992). Introducción a los métodos cualitativos de investigación, Paidós, Barcelona.

Wallerstein, Immanuel (2001). Abrir las ciencias sociales, México, Siglo XXI.

Weber, Max (1997). Economía y sociedad, México, FCE. 